\title{
Risk Assessment of Pancreatic Cysts: Benign and Malign Entwined
}

\author{
María-Victoria Alvarez-Sánchez ${ }^{1}$ Bertrand Napoléon ${ }^{2}$
}

Published online: 24 August 2020

(c) Springer Science+Business Media, LLC, part of Springer Nature 2020

The widespread availability of high-resolution cross-sectional imaging has increased the discovery rate for pancreatic cystic lesions (PCLs), a challenging entity. PCLs encompass a spectrum ranging from benign lesions to malignant disease. Though the awareness of possible malignant potential has historically led to surgical resection for their treatment, pancreatic surgery, despite recent advances, still carries significant morbidity. Therefore, the management of PCLs has transitioned toward a more selective approach with the aim of avoiding costly life-long surveillance or unnecessary burdensome surgery for benign cysts while considering appropriate surveillance for low-risk lesions and surgical treatment for cysts at high risk of current or future malignancy. Nevertheless, all diagnostic tests routinely available in clinical practice underperform in regard to differentiating benign to potentially malignant cystic tumors and to detecting high-grade dysplasia (HGD) or invasive cancer in PCLs. Several scientific societies developed clinical guidelines in order to assist physicians in the decision-making process. Guidelines from the American College of Gastroenterology (ACG), the European Study Group on Pancreatic Cysts, and the updated guidelines from the International Association of Pancreas (IAP) are the most recent and widely used [1-3]. All of these recommendations use a combination of symptoms, morphological features, and high-risk cytological features of the fine-needle aspirate in order to predict risk. Nonetheless, these guidelines are mostly based on expert opinion and supported by low-quality evidence. Every step toward improved risk stratification assessing the accuracy of established risk factors and investigating new markers

María-Victoria Alvarez-Sánchez

victoria.alvarez.sanchez@hotmail.com

1 Department of Gastroenterology, Complejo Hospitalario Universitario de Pontevedra, Instituto de Investigación Sanitaria Galicia Sur, Avenida Mourente s/n, 36071 Pontevedra, Spain

2 Department of Gastroenterology, Ramsay Générale de Santé Private Hospital Jean Mermoz, 69008 Lyon, France represents a significant advance in the clinical management of pancreatic cysts.

In this issue of Digestive Diseases and Sciences, Sun et al. analyzed clinical, biological and morphological features associated with advanced PCLs (PCLs with HGD/ invasive cancer) in a large surgical cohort of 353 patients [4]. In accordance with guidelines, the presence of mural nodules or solid components, a dilation of the main pancreatic duct (MPD) $>10 \mathrm{~mm}$, and elevated serum CA 19.9 were each independently associated with advanced PCLs, adding evidence to current recommendations. Actually, mural nodules and main pancreatic duct dilation are considered the strongest predictors independently associated with HGD and invasive cancer. A recent meta-analysis reported a high odds ratio of 9.3 for malignancy in the presence of mural nodules [5]. Moreover, several studies suggest that the mural nodule size also has a substantial role in predicting malignancy with reported cutoffs as either $>5 \mathrm{~mm}$ or $>10 \mathrm{~mm}$. Consequently, the IAP and the European guidelines adopted a threshold for surgical consideration of $\geq 5 \mathrm{~mm}[2,3]$. As demonstrated by Sun et al., endoscopic ultrasound (EUS) is the most sensitive modality for the detection of mural nodules. Owing to its high spatial resolution, EUS may detect nodules smaller than can be detected by computed tomography or magnetic resonance imaging, although it underperforms in terms of discrimination between true tissue nodules and mucus clots, which may result in needless surgery [6]. The use of contrast agents during EUS (CH-EUS) overcomes this drawback with high sensitivity and specificity by displaying vascularity in tissue nodules; therefore, $\mathrm{CH}$-EUS has been incorporated in IAP and European guidelines.

Likewise, the significant risk of malignancy risk with MPD dilation $\geq 10 \mathrm{~mm}$ found in the present study ties with current recommendations, which strongly advise surgical resection in this context. Yet, MPD dilation $\geq 10 \mathrm{~mm}$ is usually a late sign observed in a minority of cases with most studies reporting that MPD diameter of $5-9.9 \mathrm{~mm}$ is also associated with a high risk of malignancy, although with a markedly lower positive predictive value (PPV) [7], emphasizing that MPD dilation deserves further investigation. 
Accordingly, additional evaluation without immediate resection is now recommended when MPD diameter is $5-9.9 \mathrm{~mm}$ and direct surgery in cases of MPD dilation $>10 \mathrm{~mm}$ [1-3]. Nevertheless, some controversies remain regarding MPD dilation, such as how to differentiate branch-duct intraductal papillary mucinous neoplasm (BD-IPMN) with a worrisome feature from the more aggressive mixed type-IPMN or how to differentiate MPD dilation caused by direct tumor involvement from ductal hypertension secondary to mucin.

An increased level of CA 19.9 is also a predictor of advanced PCLs according to other publications. In the largest cohort thus far evaluating the utility of CA 19.9 in pathologically proven IPMN, $63 \%$ of patients with a CA 19.9>37 $\mathrm{U} / \mathrm{ml}$ had an advanced PCL [8]. Similar to any tumor marker, serum CA 19.9 should be carefully interpreted since $15 \%$ of patients with low-grade IPMN also had elevated CA 19.9, with sensitivity for advanced PCLs only $28 \%$, limiting its diagnostic power.

Although the presence of symptoms was regarded as a significant predictor of malignancy prompting surgery in the past, symptoms are now considered the least relevant marker (OR 1.6) [5], and when analyzed separately, only some of them appear to be good indicators of malignancy [9]. This discrepancy is due to nonspecific symptoms such as abdominal pain may be erroneously attributed to pancreatic cysts. In contrast, symptoms or signs specifically related to the pancreas, such as jaundice or pancreatitis, are more likely to be truly related to cysts and, in fact, are observed more frequently in patients with malignant transformation. To date, the symptom that has been most consistently associated with malignant cysts is jaundice; hence, it is regarded as a highrisk factor in guidelines leading to surgical resection [1-3, 7]. Although Sun et al. could not demonstrate jaundice as an independent predictor, jaundice was statistically associated with advanced PCLs in the univariate analysis.

Two other findings related to the cyst size and the presence of septa in the study by Sun et al. deserve further comments. A cyst size of $43.5 \mathrm{~mm}$ was identified as the optimal cutoff for differentiating between benign and malignant cysts. Although several studies in the past suggested an increased risk of malignancy for cysts $>30 \mathrm{~mm}$, later meta-analyses yielded contradictory results. Though evidence from the literature suggests that malignancy clearly correlates with larger size, some cysts $<3 \mathrm{~cm}$ may harbor a significant risk of malignancy [10]. Caution is advised when considering cyst size as a predictor of malignancy, since recent studies show that, after stratifying by other risk features, cyst size as the sole criterion is not an appropriate indication for surgery. Therefore, in the absence of other risk factors, it is now accepted that cysts $>30 \mathrm{~mm}$ can be managed conservatively under close surveillance. The IAP guidelines have shifted the size criterion from a high risk to a worrisome feature; the 2018 European guidelines have kept a threshold of $4 \mathrm{~cm}$ as a relative, but not absolute, indication for surgery $[2,3]$.

In this study, the presence of septa was an independent predictor of benign cysts. As anticipated, among benign PCLs, septa were observed in $65 \%$ of serous cystadenomas (SCNs). Almost half of the PCLs (46\%) in this series corresponded to cysts other than mucinous cysts, with $23 \%$ being SCNs. The authors emphasized that this feature has not been previously adopted in guidelines, but in fact, we cannot expect this criterion to be adopted since the presence of septa is a very common but nonspecific morphological feature of SCNs, as demonstrated in the present study, where $15 \%$ of malignant cysts also displayed septa in cross-sectional imaging.

Fourteen years have elapsed after the first guidelines were proposed in 2006, during which multiple guidelines have been released, but despite improved understanding of the natural history of pancreatic cysts and technological advances in the diagnostic field, questions remain unsolved regarding the inability to definitively discriminate between benign cysts such as SCNs and malignant or potentially malignant cysts, and even in cases where there is certainty about their premalignant potential, stratification to high or low risk for malignant transformation remains difficult. Guidelines have provided valuable support for cyst management, mainly for the risk stratification of IPMN, but they have only moderate PPV. As a result of the low diagnostic preoperative accuracy, overlooked cases of neoplasia and high rates of unnecessary surgical resections for benign or low-risk cysts still persist. Along with the discovery of new molecular markers in cyst fluid, the last decade has witnessed technological advances in the endoscopic imaging of PCLs and in cyst tissue sampling through the implementation of confocal endomicroscopy (nCLE) and throughthe-needle biopsy (TTNB), respectively. These methods are effective resources for improving the accuracy of the diagnosis and risk stratification of PCLs [6]. Nevertheless, concerns arise about the high rate of complications with TTNB, reported to be $10 \%$ in the most recent series with serious adverse events in $4 \%$ of cases and $1 \%$ mortality. In contrast, there is enough evidence showing that molecular biomarkers and nCLE safely enhance the preoperative diagnosis [11]. Although additional research on the identification of new risk factors and markers is necessary, it is now time to incorporate those that have recently been proven to be safe and effective into routine practice and clinical guidelines.

\section{Key Messages}

- Although several clinical guidelines derived from different scientific societies are available for the management of PCLs, diagnostic and risk stratification testing of PCLs 
is suboptimal, leading to a nonnegligible rate of needless surgery for benign PCLs.

- While mural nodules and main pancreatic duct dilation are the strongest morphological predictors independently associated with advanced PCLs, however, the risk of overlooked malignancy still persists.

- Further research on new patient- and cyst-related risk factors is necessary, and safe and effective recent advances such as nCLE and new molecular markers should be integrated in routine practice and clinical guidelines to improve preoperative diagnoses.

\section{Compliance with Ethical Standards}

Conflict of interest María-Victoria Alvarez-Sánchez declares no potential conflicts of interest and no financial support. Bertrand Napoléon received support from Maunakea Technologies for teaching.

\section{References}

1. Tanaka M, Fernández-Del Castillo C, Kamisawa T, et al. Revisions of international consensus Fukuoka guidelines for the management of IPMN of the pancreas. Pancreatology. 2017;17:738-753.

2. European Study Group on Cystic Tumours of the Pancreas. European evidence-based guidelines on pancreatic cystic neoplasms. Gut. 2018;67:789-804.

3. Elta GH, Enestvedt BK, Sauer BG, Lennon AM. ACG clinical guideline: diagnosis and management of pancreatic cysts. Am J Gastroenterol. 2018;113:464-479.
4. Sun L, Wang W, Zhu H, et al. High-risk characteristics associated with advanced pancreatic cystic lesions: Results from a retrospective surgical cohort. Dig Dis Sci. (Epub ahead of print). https:// doi.org/10.1007/s10620-020-06481-2.

5. Anand N, Sampath K, Wu BU. Cyst features and risk of malignancy in intraductal papillary mucinous neoplasms of the pancreas: a meta-analysis. Clin Gastroenterol Hepatol. 2013;11:913-e60.

6. Alvarez-Sánchez MV, Napoléon B. New horizons in the endoscopic ultrasonography-based diagnosis of pancreatic cystic lesions. World J Gastroenterol. 2018;24:2853-2866.

7. Del Chiaro M, Beckman R, Ateeb Z, et al. Main Duct Dilatation Is the Best Predictor of High-grade Dysplasia or Invasion in Intraductal Papillary Mucinous Neoplasms of the Pancreas. Ann Surg. 2019. https://doi.org/10.1097/SLA.0000000000003174.

8. Ciprani D, Morales-Oyarvide V, Qadan M, et al. An elevated CA 19-9 is associated with invasive cancer and worse survival in IPMN. Pancreatology. 2020;20:729-735.

9. Moris M, Raimondo M, Woodward TA, et al. Risk factors for malignant progression of intraductal papillary mucinous neoplasms. Dig Liver Dis. 2015;47:495-501.

10. Jang JY, Park T, Lee $S$, et al. Validation of international consensus guidelines for the resection of branch duct-type intraductal papillary mucinous neoplasms. Br J Surg. 2014;101:686-692.

11. Kovacevic B, Klausen P, Rift CV, et al. Clinical impact of endoscopic ultrasound-guided through-the-needle microbiopsy in patients with pancreatic cysts. Endoscopy. 2020. https://doi. org/10.1055/a-1214-6043.

Publisher's Note Springer Nature remains neutral with regard to jurisdictional claims in published maps and institutional affiliations. 